\title{
Collective excitations in electron-hole bilayers
}

\author{
G. J. Kalman ${ }^{1}$, P. Hartmann ${ }^{2}$, Z. Donkó ${ }^{2}$, K. I. Golden ${ }^{3}$ \\ ${ }^{1}$ Physics Department, Boston College, Chestnut Hill, MA 02467, USA \\ ${ }^{2}$ Research Institute for Solid State Physics and Optics of the Hungarian Academy of Sciences, \\ H-1525 Budapest, P.O. Box 49, Hungary and \\ ${ }^{3}$ Department of Mathematics and Statistics and Department of Physics, \\ University of Vermont, Burlington, VT 05401, USA
}

(Dated: September 3, 2018)

\begin{abstract}
We report a combined analytic and Molecular Dynamics analysis of the collective mode spectrum of an electron-hole (bipolar) bilayer in the strong coupling quasi-classical limit. A robust, isotropic energy gap is identified in the out-of-phase spectra, generated by the combined effect of correlations and of the excitation of the bound dipoles; the in-phase spectra exhibit a correlation governed acoustic dispersion for the longitudinal and transverse modes. Strong nonlinear generation of higher harmonics of the fundamental dipole oscillation frequency and the transfer of harmonics between different modes is observed. The mode dispersions in the liquid state are compared with the phonon spectrum in the crystalline solid phase, reinforcing a coherent physical picture.

PACS numbers: PACS: 52.27.Gr, 52.65.Yy, 73.21.-b, 73.20.Mf
\end{abstract}

Electron/hole bilayer systems consisting of a pair of two dimensional (2D) layers of electrons and holes of equal density, (bipolar bilayers -BPBL-s) have attracted a great deal of attention over the past decade [1]. The main feature of the system is that in the appropriate parameter domain, the electrons and the holes bind to each other in a dipole-like excitonic formation 1,2]. These excitons may also form a Bose condensate [1, 3, 4, 5]. It is also expected that at strong enough coupling the system undergoes a transition into a Wigner crystal-like solid phase [2, 3]. A recent work has also pointed out the exciting possibility of the existence of a supersolid phase over the dipole-solid domain 6]. A study of a strongly coupled classical BPBL, based on Monte Carlo simulation 2] revealed the existence of dipole-liquid, dipole-solid, Coulomb-liquid and Coulomb-solid "phases", depending on the value of the coupling parameter and the layer separation $d$. While the details of the boundaries and transitions between the different phases would be different in the classical and quantum domains, there is little doubt that the topology of the classical phase diagram is quite generally correct [2].

The problem we address in this Letter is the collective mode spectrum of the BPBL in the strong coupling (SC) regime, in a parameter range spanning all four phases but focusing on the more important and more intriguing SC liquid state. The question of affinity of this spectrum with that of the electronic bilayer (EBL), where the charges in the two layers are identical, arises; this latter system is now fairly well understood [7, 8, [9]. From the point of view of the collective mode spectrum, the dominant feature of both systems in the SC regime is the localization or quasilocalization of the particles, either in the crystalline solid or in the SC liquid phase 10]. This fact and the physical separation of the two oppositely charged layers that prevents their collapse, allows one to represent the dynamics of the collective modes in the BPBL, even in the quantum domain, through a classical mod- eling [7, 10, 11]. The in-layer exchange effects, precisely because of the quasilocalization of the charges, contribute only to generating an exchange-correlation energy, which is well emulated by the equivalent classical correlation energy 12], and tunneling is insignificant, because for any reasonable layer separation $d$ value, $d / a_{B} \gg 1$. This latter condition also ensures that the excitation energy of the exciton is well described in terms of its classical Kepler frequency.

Our approach is based on a combined classical Molecular Dynamics (MD) simulation and theoretical analysis. To study collective excitations, simulation of a large assembly of particles is needed, which, at present, becomes feasible only by using a classical approach. What the classical approach obviously misses, however, is the effect of condensate on the dispersion. This is further discussed in our conclusions.

While the hole and electron masses are, generally speaking, unequal, a simplified symmetric model with equal masses gives insight into the dominating aspects of the mode spectrum. Within the classical modeling, the symmetric BPBL system is then characterized by (i) the in-layer Coulomb coupling coefficient $\Gamma=\left(e^{2} / a k T\right)$ $\left[a=(n \pi)^{-1 / 2}\right.$ is the Wigner-Seitz (WS) radius, $n$ is the areal density of particles], and (ii) the separation of the two layers $d$. Addressing first the theoretical description of the collective mode spectrum, one should realize that, in contrast to the case of the EBL, a perturbative approach is bound to fail - even at low $\Gamma$ values - due to the dominance of the excitonic bound state in the spectrum. Nevertheless, as a matter of orientation, we can start with the Random Phase Approximation (RPA) for the mode spectrum. The interaction between the particles can be characterized by the matrix $\varphi_{11}(\mathbf{k})=\varphi_{22}(\mathbf{k})=2 \pi e^{2} / k$, $\varphi_{12}(\mathbf{k})=\varphi_{21}(\mathbf{k})=-\left(2 \pi e^{2} / k\right) \exp (-k d)$. Its diagonalization in the layer space leads to the identification of 2 longitudinal modes as in-phase and out-of-phase $( \pm)$ modes: $\omega_{ \pm}^{L}(k)=\omega_{0} \sqrt{k a}[1 \mp \exp (-k d)]^{1 / 2}$, where 
$\omega_{0}=\sqrt{2 e^{2} / m a^{3}}$. The small- $k$ behavior of the + mode is acoustic, here with a slope $s_{+}=\omega_{0} \sqrt{a d}$, while the mode has the typical 2D $\omega \propto \sqrt{k a}$ behavior. Thus, the RPA misses, as it must, the appearance of the frequency of the intrinsic oscillation (excitation) of the dipole in the spectrum. In addition, the RPA it also unable to account for the SC collective effect, in particular, the generation of transverse shear modes. In an appropriate SC description, both of these effects should be correctly represented. The approach we adopt is the Quasilocalized Charge Approximation (QLCA) 10, 13], the method that we previously employed to identify the gapped excitation in the collective spectrum of the EBL [7]. The QLCA approach has also been applied to a variety of Coulombic and other systems in the SC liquid state 14], with results that have been successfully tested against the outcomes of computer simulations $[8,9]$ and laboratory experiments 12, 15, 16 in both the classical and quantum domains. The longitudinal $(L)$ and transverse (T) QLCA dielectric matrices in layer space $(i, j)$ [7]

$$
\varepsilon_{i j}^{L, T}(\mathbf{k}, \omega)=\delta_{i j}-\frac{n k^{2}}{m} \varphi_{i l}(\mathbf{k}) \cdot\left[\omega^{2} \mathbf{I}-\mathbf{D}^{L, T}(\mathbf{k})\right]_{l j}^{-1},
$$

become functionals of the longitudinal $(L)$ and transverse $(T)$ projections of the dynamical matrix $D_{i j}^{\mu \nu}(\mathbf{k})=$ $\frac{1}{m A} \sum_{\mathbf{q}} q^{\mu} q^{\nu} \varphi_{i j}(q)\left[h_{i j}(|\mathbf{k}-\mathbf{q}|)-\delta_{i j} \sum_{l} \varphi_{i l}(q) h_{i l}(\mathbf{q})\right]$, $h_{i j}(\mathbf{q})$ are the Fourier transforms of $h_{i j}(r)=g_{i j}(r)-1$, where $g_{i j}(r)$ is the pair distribution function and $A$ is a large but finite surface. The resulting mode structure

$$
\begin{array}{r}
\omega_{ \pm}^{L}(\mathbf{k})=\left[\omega_{0}^{2} k a(1 \mp \exp (-k d))+D_{11}^{L}(\mathbf{k}) \pm D_{12}^{L}(\mathbf{k})\right]^{1 / 2}(2) \\
\omega_{ \pm}^{T}(\mathbf{k})=\left[D_{11}^{T}(\mathbf{k}) \pm D_{12}^{T}(\mathbf{k})\right]^{1 / 2}(3)
\end{array}
$$

now exhibits a behavior significantly different from its RPA counterpart. These dispersion curves are displayed in Fig. 1 together with MD simulation results (see below).

(i) At $k=0$ the - mode becomes a gapped mode (see also Fig. 2a), with

$$
\omega_{-}^{L}(k=0)=\omega_{-}^{T}(k=0) \equiv \Omega_{G}(d)
$$

where $\Omega_{G}(d)$ is a functional of $h_{12}(r)$ as given in [17]. In the BPBL $h_{12}(r)$ is governed by a central peak $h_{12}^{\prime}(r)$ around $r=0$ [2] (see Fig. 2r). For $d / a<1 \Omega_{G}(d)$ is well described by replacing $h_{12}(r)$ by $h_{12}^{\prime}(r)$. When this central peak is approximated by a Gaussian (representing a thermally excited dipole) $\Omega_{G}(d)$ becomes $\Omega_{K}^{\prime}(d)$, the thermally broadened small amplitude oscillation Kepler frequency $\Omega_{K}(d)=\sqrt{2 e^{2} / d^{3} m}=\omega_{0}(a / d)^{3 / 2}$ of the oscillating dipole. Dipole-dipole correlations shift $\Omega_{G}(d)$ from this value only for $d / a>1$.

(ii) The + mode may be regarded as a density oscillation of the dipoles: with a $1 / r^{3}$ type dipole-dipole interaction, this suggests an $\omega \propto k$ dispersion. This is borne out by the present calculation: the + mode shows an acoustic behavior, similarly to the RPA result, but with a phase velocity instead of being proportional to

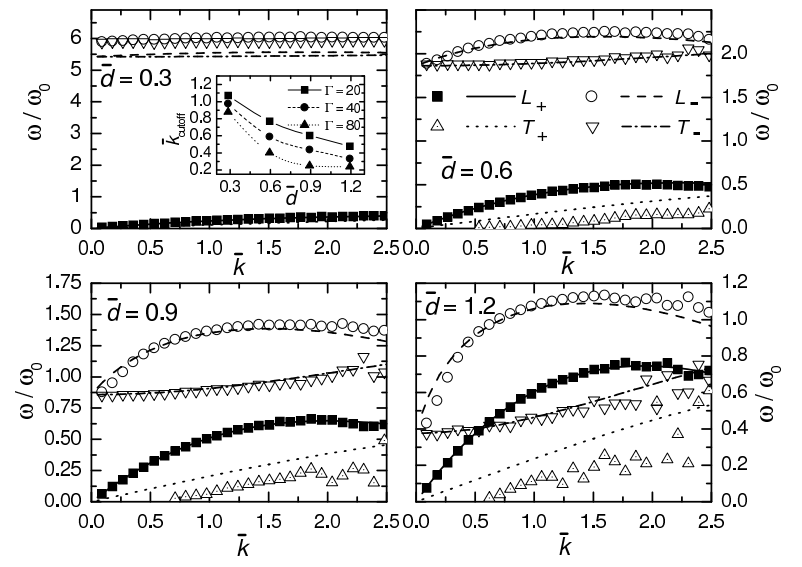

FIG. 1: Dispersion relations for the $L_{+}, L_{-}, T_{+}$, and $T_{-}$ modes $(\bar{k}=k a)$ at $\Gamma=40$ and different layer separations. The legend shown in the $\bar{d}=d / a=0.6$ plot applies to all panels. Lines: QLCA, symbols: MD simulation. Inset shows the observed values of $\bar{k}_{\text {cutoff }}$ of the $T_{+}$mode.
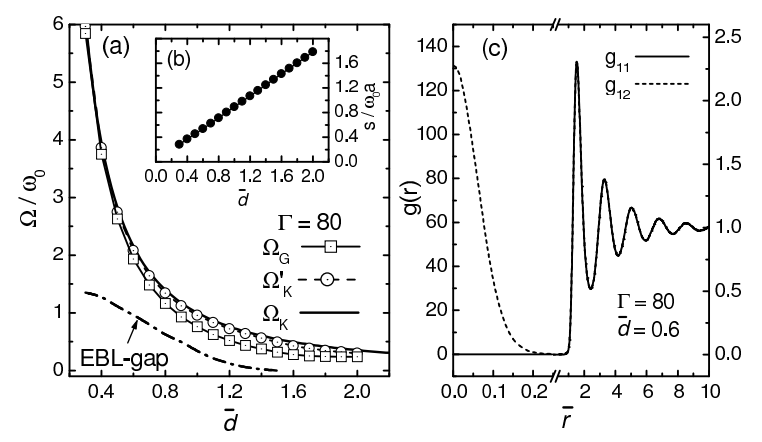

FIG. 2: (a) Dependence of the Kepler frequency $\Omega_{K}$, the thermally shifted Kepler frequency $\Omega_{K}^{\prime}$ and the gap frequency $\Omega_{G}$ on $\bar{d}$ at $\Gamma=80$; also shown for comparison is the EBL gap. (b) $s_{+}$sound velocity vs. $\bar{d}$ for $\Gamma=80$. (c) MD pair correlation functions for $\Gamma=80$ and $\bar{d}=0.6$ (left and right vertical scales belong to the left and right horizontal parts).

$(d / a)^{1 / 2}$, is of the order of $d / a: s \equiv \omega_{+}^{L}(k \rightarrow 0) / k=$ $\omega_{0} d\left[(99 / 96) \int \mathrm{d} \bar{r} g_{12}(r) / \bar{r}^{2}\right]^{1 / 2}$ (see Fig. [2]).

(iii) In addition to the 2 longitudinal modes 2 transverse shear modes appear, with a behavior qualitatively similar to their longitudinal counterparts, except that, as expected for shear modes in a liquid, the $T_{+}$mode do not extend below a finite $k_{\text {cutoff value (see inset in Fig. (1) }}$ [18.

(iv) For $k \rightarrow \infty$ all four modes approach the Einstein frequency (the frequency of oscillation of a single particle in the frozen environment of the others) [7] of the system:

$$
\Omega_{E}^{2}(d)=\Omega_{E, 2 D}^{2}+\frac{1}{2} \Omega_{G}^{2}(d),
$$

where $\Omega_{E, 2 D}^{2}=\left(\omega_{0}^{2} / 2\right) \int \mathrm{d} \bar{r} g_{11}(r) / \bar{r}^{2} \cong 0.38 \omega_{0}^{2}$ is the Einstein frequency of an isolated 2D layer [14] (see later).

Illustrative longitudinal and transverse current-current correlation spectra obtained from our MD simulations 

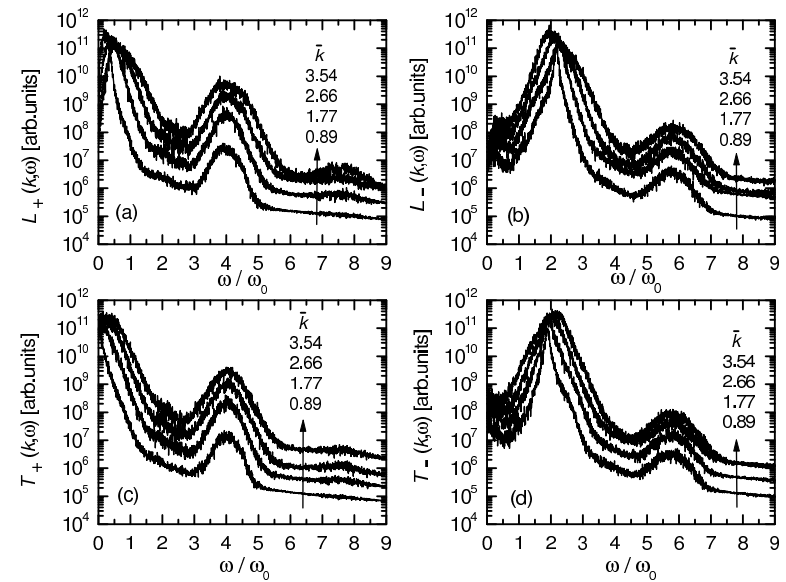

FIG. 3: Longitudinal current $(\mathrm{a}, \mathrm{b})$ and transverse current (c,d) fluctuation spectra obtained at $\Gamma=40$ and $\bar{d}=0.6$. The arrows indicate increasing wave numbers $\bar{k}$ as listed in the panels. Note the appearance of higher harmonics of the gap frequency: the second and fourth harmonic in the + spectra, and the third harmonic in the - spectra.

(based on the Particle-Particle Particle-Mesh (PPPM) method [19] with periodic boundary conditions) are displayed in Fig. 3 for $\Gamma=40$ and $d / a=0.6$. Collective modes show up as peaks in the correlation spectra. The MD-generated dispersion relations inferred from the spectral peaks for the 4 modes for $\Gamma=40$ and for a series of $d / a$ values are displayed in Fig. 1 together with theoretically calculated values. Agreement between the QLCA theory and simulation data is very good, especially for lower $k$ values. Deviation from the characteristic $d^{3 / 2}$ behavior of $\Omega_{K}(d)$ seems to occur on passing the (dipole liquid)/(Coulomb liquid) phase boundary [2]. Comparison with the EBL spectrum [7, 8] reveals a more pronounced and more robust gap value (Fig. 22 $\mathrm{k}$ ), which is not unexpected if one is mindful of the different physical mechanisms responsible for creating the gap.

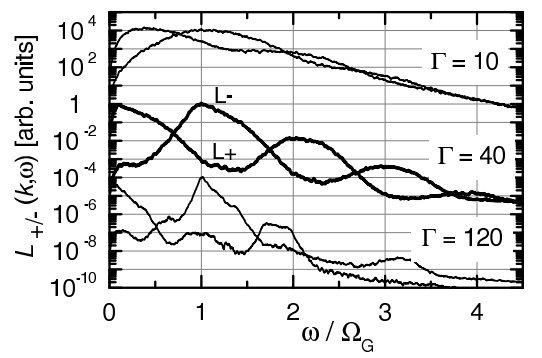

FIG. 4: Longitudinal current fluctuation spectra showing the higher harmonics of the gap frequency for $\bar{k}=3.54, \bar{d}=0.6$, $\Gamma=10,40$ and 120 . The transverse spectra exhibit similar behavior.

A remarkable feature of the simulation spectra is the appearance of higher harmonics of the gap frequency: we observe the emergence (a) of the third harmonic in the - spectra and (b) of the second harmonic (but not the fundamental) in the + spectra) [Fig. 圆. These harmonics are the most pronounced around $\Gamma=40$ in the strongly coupled dipole liquid phase, showing a diminishing trend both for lower and higher coupling values: at high $\Gamma$ values, the low amplitude of the thermally excited oscillations is not conducive to the generation of harmonics, while at lower $\Gamma$ values, the thermal motions damp the higher harmonics. The analysis of the details of the nonlinear processes underlying the harmonic generation will be presented elsewhere.

We calculate the phonon spectrum of the crystalline phase, through the standard harmonic approximation, by summing over a lattice of $2 \times 10^{7}$ sites. In the solid phase the BPBL crystallizes in a hexagonal structure, with the particles in the two layers facing each other (for any layer separation, in contrast to the EBL which exhibits a variety of lattice structures). One can recognize the 4 modes identified in the liquid state, with an expected anisotropic dispersion and with the understanding that the "longitudinal" and "transverse" labeling of the modes describes their polarization for propagation along the principal crystal axes only.
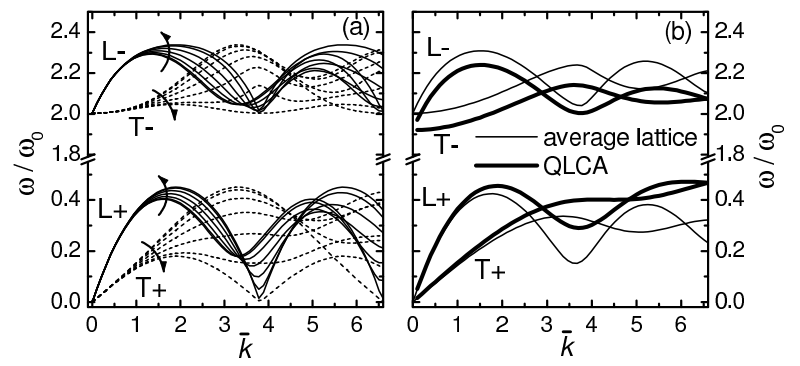

FIG. 5: (a) Lattice dispersion of the quasi- $L_{+}$and quasi- $L_{-}$ (full lines) and quasi- $T_{+}$and quasi- $T_{-}$(dashed lines) modes for a series of lattice angles between $0^{\circ} \leq \alpha \leq 30^{\circ}(\alpha=$ $0^{0}$ corresponds to the nearest neighbor direction, the arrows indicate increasing $\alpha$ ). (b) Comparison at $\bar{d}=0.6$ of QLCA (at $\Gamma=80)$ and angular averaged lattice dispersion.

The mode spectrum is shown in Fig. [5 For the sake of comparison with the QLCA dispersion, also shown are the angle-averaged longitudinal and transverse projections: the similarity between the two is the consequence of the underlying microscopic order in the SC liquid as predicted by the QLCA. The - modes in the lattice spectrum exhibit a $k=0$ gap, very similar to the one in the liquid spectrum; in contrast to the EBL, the gap is not split by the lattice anisotropy. This is a consequence of the quasi-isotropy of the hexagonal lattice environment to $O\left(k^{2}\right)$. Physically, the - modes can be regarded, in a good approximation, especially for small $d / a$ values, as the superposition of a + mode on the $\Omega_{K}$ dipole oscillation frequency. The angle-averaged modes emulate the liquid-phase mode dispersion for low and moderate $k$-values, but deviate substantially from it for $k \rightarrow \infty$ (see Fig. 5b). To understand this difference, one has to focus on $h_{12}^{\prime}(r)$, which shows a quasi-Gaussian behavior in the liquid, but becomes a delta-function in a 
perfect $T=0$ lattice. The contribution of oscillating dipoles within a Gaussian distribution results in a superposition of random phases at $k \rightarrow \infty$, averaging out to 0, ensuring the limit shown in Eq. 5 . In contrast, the delta-function contribution generates additional coherent $k$-independent terms that survive for $k \rightarrow \infty$. The simulation results clearly indicate that even for high $\Gamma$ values the large but finite $k$ dispersion curves follow the trend set by Eq. 5] describing the liquid, rather than the one corresponding to the lattice. The great sensitivity of the behavior of the mode dispersions at higher $k$ values to the width of $h_{12}^{\prime}(r)$ could motivate an observational technique for acquiring information of the width of central peak distribution through tracking the high- $k$ behavior of the collective excitations.

The phonon spectrum in the BPBL was the subject of a recent investigation by Ref. 21]. This work addressed the behavior of the dipolar "phase", within an approximation geared to the assumed separability of the overall collective and intrinsic dipolar excitations: our results show that this technique, reasonable as it may appear, leads to an unphysical behavior in the calculated mode dispersion. In particular, 21] finds (i) that the gap is split and there are distinct transverse and longitudinal gap values; (ii) that both of these exhibit an anisotropic behavior; (iii) that for $k \rightarrow 0$ the - mode dispersion curves exhibit a negative slope; and (iv) that the $k \rightarrow \infty$ behavior does not conform to that demanded either by Eq. [5 or by the lattice condition. These features violate basic physical principles pointed out above.

In summary, we have determined the dispersion characteristics of a strongly coupled symmetric bipolar (electron/hole) bilayer in the strongly coupled domain, where the dominant exchange-correlation energy provides a sufficient quasilocalization of the particles to engender a quasi-classical behavior. Our analysis in the strongly coupled liquid phase is based on the MD simulation of the density and current fluctuation spectra and on the application of the theoretical QLCA technique. The benchmark phonon spectrum in the crystalline solid phase is determined through lattice summation technique. The results of all these various approaches reinforce each other and provide a coherent physical picture. The 4 modes, characteristic of bilayer systems (in-phase/out-of-phase, longitudinal/transverse) emerge, with the expected $k=0$ energy gap in the spectrum of the out-of-phase mode 7, 8]. The latter, is now mostly governed by the intrinsic oscillation frequency of the dipoles and affected by collective interaction only in the Coulomb phase, for $d \gtrsim a$. A remarkable effect, unique to the strongly coupled liquid phase, the generation of harmonics of the gap frequency and the transfer of the even harmonics from the out-of-phase mode to the in-phase mode, has been observed.

The presence of the condensate would manifest itself primarily by reducing the quasi-localization of the particles (as clearly demonstrated by 22]). A rough estimate of the degree of this reduction is provided by replacing $h(k)$ (and thus $D(k))$ by $\bar{h}(k)=(1-f)^{2} h(k)$, where $f$ is the condensate fraction, as suggested by [23]. Since $f$ in the SC regime is expected to be quite small ( $f=0.02$ 5]), this should not be a dramatic effect. In the solid phase, if the supersolid develops, the system would probably behave as a binary mixture of the normal crystal lattice and the superfluid liquid constituted by the vacancies [24], with a coupling between the lattice phonons and the collective excitations of the latter. These issues have to be addressed elsewhere.

This work was supported by NSF Grants PHY0514619 (GJK), PHY-0514618 (KIG), and by the Hungarian Fund for Scientific Research and the Hungarian Academy of Sciences, OTKA-T-48389, MTA-OTKA90/46140, OTKA-PD-049991.
[1] P. B. Littlewood and X. Zhu, Phys. Scr. T 68, 56 (1996); U. Sivan et al, Phys. Rev. Lett. 68, 1196 (1992).

[2] P. Hartmann et al, Europhys. Lett. 72 (3) 396 (2005).

[3] Yu. E. Lozovik and V. I. Yudson, Pis'ma Zh. Eksp. Teor. Fiz. 22, 556 (1975) [JETP Lett. 22, 274 (1975)]; Solid State Commun. 19, 391 (1976); T. Fukuzawa et al, Phys. Rev. Lett. 64, 3066 (1990).

[4] P. Ludwig et al, phys. stat. sol. (2006); A. V. Filinov et al, phys. stat. sol. (2003); J. E. Golub et al, Phys. Rev. B 41 8564(R) (1990).

[5] S. De Palo et al, Phys. Rev. Lett. 88, 206401 (2002).

[6] Y. N. Joglekar et al, ArXiv:cond_mat/0606124v1

[7] G. Kalman et al, Phys. Rev. Lett. 82, 3124 (1999).

[8] Z. Donkó et al, Phys. Rev. Lett. 90, 226804 (2003).

[9] S. Ranganathan and R. E. Johnson, Phys. Rev. B 69, 085310 (2004).

[10] K. I. Golden and G. J. Kalman, Phys. Plasmas 7, 14 (2000); 8, 5064 (2001).

[11] Z. Donkó et al, Phys. Plasmas 10, 1563 (2003).

[12] K. I. Golden et al, Phys. Rev. E 70, 026406 (2004).
[13] G. Kalman and K. I. Golden, Phys. Rev. A 41, 5516 (1990).

[14] K. I. Golden et al, Phys. Rev. A 46, 3454 (1992); 3463 (1992).

[15] C. F. Hirjibehedin et al, Phys. Rev. B 65, 161309 (R) (2002).

[16] A. Piel et al, Phys. Plasmas 13, 042104 (2006).

[17] K. I. Golden et al, Phys. Rev. E 71, 036401 (2005).

[18] M. S. Murillo, Phys. Rev. Lett. 85, 2514 (2000); V. Nosenko et al, Phys. Rev. Lett. 97, 115001 (2006).

[19] R. W. Hockney and J. W. Eastwood, Computer Simulation Using particles (McGraw-Hill, New York, 1981).

[20] G. J. Kalman et al, Phys. Rev. Lett. 92, 065001 (2004).

[21] D. V. Kulakovskii et al, JETP 99, 850 (2004).

[22] J. B. Mayers, Phys. Rev. B 64, 224521 (2001).

[23] G. J. Hyland et al, Phys. Lett. A 31A, 465 (1970).

[24] N. Prokofev and B. Svistunov, Phys. Rev. Lett. 94, 155302 (2005). 